\title{
A Síntese Verde - (1) Conceito e Génese
}

\author{
Adélıo A. S. C. Machado*
}

\begin{abstract}
$D$ iscute-se o conceito de Síntese Verde (SV) e a sua génese, procurando caracterizar-se a Síntese Verde ideal e analisar as dificuldades da sua implementação. Finalmente, põe-se em evidência as ideias inovatórias no domínio da química de síntese que fizeram emergir o conceito moderno de SV nos inícios da década de noventa do século passado.
\end{abstract}

A concepção de vias de síntese, a par do design das moléculas dos novos compostos a preparar, são as actividades mais fulcrais da Química Verde (QV). Uma análise da via de síntese no contexto da QV revelou vários aspectos interessantes, dois dos quais serão abordados nesta sequência de dois artigos, que têm globalmente dois objectivos principais. O primeiro é apresentar e discutir o conceito de Síntese Verde (SV) como o alvo mais importante para implementação da Química Verde $(\mathrm{QV})$ pelos químicos académicos. O segundo é mostrar que tem vindo a ser usada pela Indústria Química quase desde a sua emersão, estando a SV historicamente associada ao processo contínuo usado pela Química Industrial para o fabrico dos compostos de base em larga escala - certas características de verdura da síntese que implicam simplicidade química, nomeadamente a boa utilização dos átomos e o uso de reacções catalíticas, foram importantes na génese e desenvolvimento deste tipo de processo de fabrico.

Para cumprir o primeiro destes objectivos, neste artigo começa por apresentar-se o conceito de via de síntese, já que este é quase ignorado no ensino da química académica, e exemplifica-se como as suas características afectam a verdura da síntese.

\footnotetext{
* Departamento de Química da Faculdade de Ciências da Universidade do Porto, R. Campo Alegre, 687, Porto E-mail: amachado@fc.up.pt
}

Depois, caracteriza-se o conceito de SV ideal, discutindo-se as respectivas propriedades desejáveis, e exemplifica-se as razões por que a SV real fica quase sempre longe da ideal. Finalmente, apresenta-se brevemente os avanços de conhecimento químico que conduziram à génese da SV, muito ligada à da própria QV. O segundo objectivo acima será cumprido no artigo seguinte.

\section{Via de Síntese}

Embora o ensino da química seja feito predominantemente com base em reacções químicas individualizadas, na prática industrial, os processos de fabrico de compostos envolvem em geral uma sucessão de reacções químicas: sucede quase sempre que a reacção usada como cerne do processo tem de ser precedida e seguida por outras reacções, isto é, é englobada numa via (ou rota) de síntese mais ou menos complexa (ver a Figura 1). Esta situação é inevitável porque não se consegue passar directamente dos reagentes disponíveis para o produto requerido e se tem de preparar um ou mais compostos intermediários até se chegar a ele, por meio de reacções sucessivas. Em consequência, as vias de síntese envolvem geralmente maior complexidade do que sugere o ensino académico, reducionista, da química - o que implica que, frequentemente, a obtenção do produto seja acompanhada por grande quantidade de resíduos, muito superior à dele.
Frequentemente, as vias de síntese concebidas pelos químicos académicos não são adequadas para a prática industrial, pelo que uma primeira etapa do desenvolvimento do processo industrial para fabrico de um composto consiste na escolha, revisão e optimização de uma síntese académica com vista a obter uma síntese adequada para ser realizada em grande escala - em que é importante atender, nomeadamente, à exequibilidade técnica e económica, essenciais na actividade industrial. Esta tarefa, realizada por equipas que integram químicos de processo e engenheiros químicos, é geralmente muito complexa e trabalhosa e não vai ser discutida aqui (embora seja muito importante - ver, por exemplo, [1-10]1). A seguir apresentam-se apenas alguns conceitos básicos relevantes para permitir a compreensão das secções seguintes, e que façam sentir, em particular, por que uma concepção adequada da via de síntese é essencial para obter verdura.

No que respeita à implementação prática, a complexidade de uma via de síntese não depende apenas do número e natureza das reacções químicas que a constituem, mas também de vários outros factores operacionais, por exemplo:

(i) reacções consecutivas poderem, ou não, ser realizadas conjuntamente, de uma sóvez, em grupos; e

(ii) haver, ou não, necessidade de realizar operações físicas entre as reacções (separações, purificações, etc.). 


\title{
SÍNTESE EM QUÍMICA FINA
}

\section{VIA DE SÍNTESE COM PASSOS MÚLTIPLOS}

\author{
$R_{i}$ REAGENTES \\ I COMPOSTOS INTERMÉDIOS \\ P PRODUTO
}

$\mathbf{R}_{1} \mathbf{R}_{2} \rightarrow \mathrm{I}_{1}\left(+\mathbf{R}_{3}\right) \rightarrow \mathrm{I}_{2}\left(+\mathbf{R}_{4}\right) \rightarrow \mathrm{I}_{3}\left(+\mathbf{R}_{5}\right) \rightarrow \ldots \rightarrow \mathbf{I}_{4}\left(+\mathbf{R}_{6}\right) \rightarrow \mathrm{P}$

PASSOS

$$
\begin{gathered}
\mathrm{R}_{1}+\mathrm{R}_{2} \rightarrow \mathrm{I}_{1}+\mathrm{A} \\
\mathrm{I}_{1}+\mathbf{R}_{3} \rightarrow \mathrm{I}_{2}+\mathrm{B} \\
\mathrm{I}_{2}+\mathbf{R}_{4} \rightarrow \mathrm{I}_{3}+\mathrm{C} \\
\mathrm{I}_{3}+\mathbf{R}_{5} \rightarrow \mathrm{I}_{4}+\mathrm{D} \\
\quad \ldots \\
\mathrm{I}_{4}+\mathbf{R}_{6} \rightarrow \mathrm{P}+\mathrm{E}
\end{gathered}
$$

\section{EQUAÇÃO GLOBAL:}

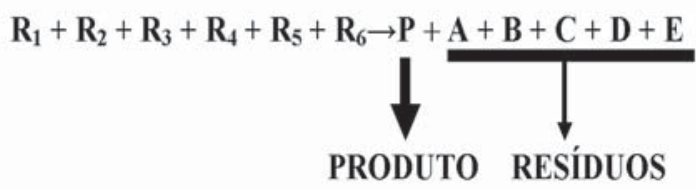

Figura 1 - Via de síntese: os processos químicos que envolvem vias sintéticas com muitos passos reaccionais com reagentes estequiométricos produzem quantidades relativamente elevadas de resíduos

Em princípio, a complexidade da realização prática da via de síntese será tanto menor quanto mais se puder agrupar reacções para serem realizadas em conjunto e quanto menos operações físicas de isolamento e purificação de intermediários forem necessárias entre as reacções consecutivas - neste caso, poupa-se meios de produção variados (ver adiante). Por isso, para aferir a complexidade/ simplicidade das vias de síntese usase, além do número de reacções, dois conceitos que interessa considerar: passo e etapa. O número e os tipos de passos e a sua agregação em etapas influenciam marcadamente a complexidade operacional da via de síntese e os recursos requeridos por ela.

Passo. Define-se passo químico como sendo uma reacção que provoca uma mudança estrutural na molécula e origina um produto isolável - que, no entanto, pode não ser preciso separar, de facto, para continuar a realizar a via de síntese ${ }^{2}$. O isolamento de um composto significa tempo, equipamento, trabalho, etc., enfim, custos adicionais, pelo que, na prática industrial, sempre dominada pelo controlo de custos, se se puder dispensar, não se faz. Quando se fazem comparações de vias de síntese, a via com um menor número de passos químicos poderá ser concretizada mais facilmente - se bem que seja preciso atender também à complexidade dos passos individuais.

O termo passo de purificação referese a operações de separação e tratamentos destinados a aumentar a pureza de um reagente intermediário ou do produto final (destilações, recristalizações, etc.). Os passos de purificação distinguem-se dos passos químicos porque, ao contrário destes, não envolvem ruptura e formação de ligações químicas.

Etapa. Por outro lado, define-se etapa como uma série de operações constituídas por um ou mais passos, químicos e de purificação, seguidas de isolamento de um composto como sólido, líquido ou em solução, que pode ser um reagente intermediário ou o pro- duto final - entre as sucessivas reacções e operações de uma etapa não é necessário isolar compostos (na Figura 2 [11] exemplifica-se a diferença entre passo e etapa). Na prática, o número de etapas de uma via de síntese é o número de compostos que são isolados ao longo dela. Por exemplo, quando os compostos intermediários e final forem todos sólidos, a obtenção de cada sólido conclui uma etapa e o número de etapas da via de síntese é o número de sólidos isolados.

Estratégia de agregação de passos em etapas. Do ponto de vista da realização prática dos processos, em princípio, há vantagens em concentrar o mais possível os passos em etapas (ver exemplo na Figura 3) - poupa naturalmente trabalho, tempo de reactor, equipamento e outros recursos materiais, por exemplo, solventes e reagentes auxiliares, o que se traduz em desmaterialização da via e consequente aumento da sua verdura. No entanto, nem sempre esta é a melhor atitude, porque pode conduzir a etapas demasiado complexas, por exemplo por a sua execução ser difícil de controlar ou exigir equipamento específico. Neste caso, é melhor não sacrificar demasiadamente a simplicidade, apesar de esta ser um objectivo sempre a ter em conta, muito em particular na actividade industrial - por exemplo, se uma agregação de passos provocar uma deterioração intolerável da qualidade do composto obtido no fim da etapa, é preferível "dar os passos" em etapas separadas.

Protecção de grupos funcionais. Frequentemente, nas sínteses das moléculas orgânicas complexas que constituem a Química Industrial Fina, para obter selectividade, é necessário proteger parte dos grupos funcionais, quando se pretende realizar transformações noutros por meio de reacções químicas adequadas. Para isso, introduzem-se, mediante reacções adicionais, grupos protectores que se liguem aos grupos a conservar e os bloqueiem aquando da reacção nos grupos funcionais a transformar - isto é, que os protejam de modo a que não reajam. Depois de concretizada a transformação pretendida em outros locais da molécula, eliminamse os grupos protectores dos grupos 
bloqueados mediante reacções adicionais convenientes (ver exemplo na Figura 4 [12], em que um dos grupos cetona é protegido por reacção com etanodiol antes de se actuar sobre o outro). Este procedimento lida com os grupos funcionais de forma individual - considera-os e protege-os um a um - pelo que, na síntese de moléculas complexas, significa muito trabalho e dispêndio elevado de reagentes complementares, usados nas protecções.

As técnicas de protecção de grupos funcionais desenvolveram-se muito nos últimos cinquenta anos e passaram a ser invariavelmente usadas, por exemplo, na síntese total ${ }^{3}$ de compostos de origem bioquímica realizada no laboratório, cujas moléculas são frequentemente muito complexas e, posteriormente, foram adoptadas rotineiramente mesmo na síntese de moléculas menos complexas. Aliás, a protecção de grupos funcionais, embora tivesse permitido a síntese de muitos compostos, foi responsável pelo aparecimento de um novo pesadelo dos químicos orgânicos de síntese: em certos casos, depois de chegar à molécula desejada, mas ainda protegida, tiveram a surpresa desagradável de não conseguir remover os grupos protectores - porque estes estavam teimosamente agarrados à molécula e resistiam à separação. Pior ainda, por vezes, quando os químicos intensificavam a agressividade do processo de remoção, este acabava por destruir a molécula, fazendo-os perder o trabalho que podia ter demorado muitos meses a realizar!

No entanto, mesmo que não ocorram desastres deste tipo, cada protecção significa sempre, pelo menos, duas reacções adicionais na via de síntese (uma de protecção, outra de desprotecção) e envolve reagentes auxiliares estequiométricos, que podem ou não ser recuperados e reciclados no desbloqueamento. Por outro lado, a protecção/desprotecção é frequentemente responsável por um abaixamento do rendimento da síntese, que pode ser acentuado quando, por exemplo, a remoção se mostra difícil ou ocorrem reacções laterais indesejadas nos grupos protectores. Em suma, o "andar para atrás e para a frente" do resguardo/desbloqueamento de gru-

\section{PASSO E ETAPA}

\section{1}

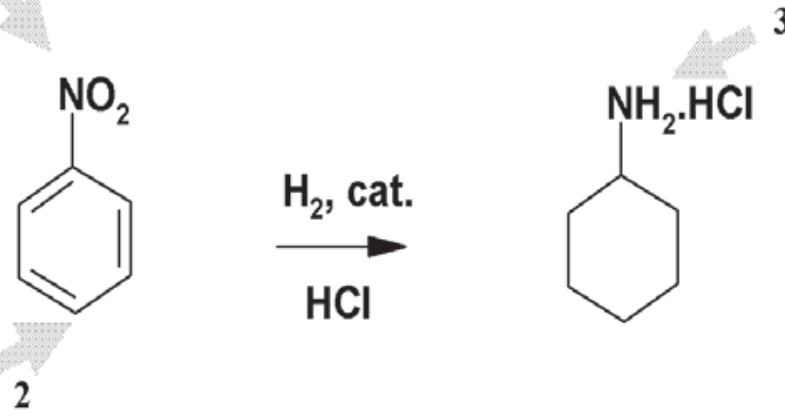

Figura 2 - Diferença entre passo e etapa: a transformação de nitrobenzeno em cloreto de ciclohexanoamónio pode ser realizada numa única etapa mas consta de três passos químicos: (1) redução do grupo nitro; (2) redução do anel aromático; e (3) formação do sal ónio

\section{AGREGAÇÃO DE PASSOS EM ETAPAS}

\begin{tabular}{|c|c|c|c|c|c|c|c|c|}
\hline 1 & 2 & 3 & 4 & 5 & 6 & 7 & 8 & 9 \\
\hline $\mathbf{P P}_{1}$ & $P Q_{1}$ & $\mathrm{PQ}_{2}$ & $\mathrm{PQ}_{3}$ & $\mathbf{P P}_{2}$ & $P Q_{4}$ & $\mathrm{PQ}_{5}$ & $\mathbf{P P}_{3}$ & $\mathbf{P P}_{4}$ \\
\hline 1 & & & $\Gamma$ & & & $T$ & & \\
\hline $\mathbf{E}_{1}$ & & & 2 & & & $\mathbf{E}_{3}$ & & $\mathbf{E}_{4}$ \\
\hline
\end{tabular}

PP $_{\mathrm{i}} \quad$ PASSO DE PURIFICAÇÃO

PQ $_{i} \quad$ PASSO QUÍMICO

$E_{i} \quad$ ETAPA

Figura 3 - Importância da agregação de passos em etapas: a agregação representada permite realizar o processo de 9 passos em 4 etapas - o que só exige 4 unidades de equipamento

\section{USO DE GRUPO PROTECTOR}
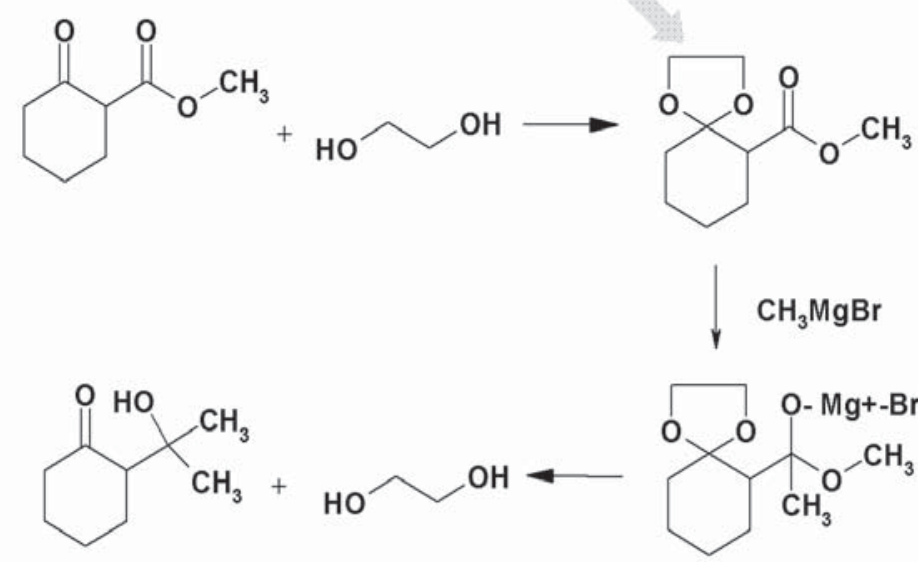

Figura 4 - O uso de grupos protectores complica a síntese: o etanodiol é usado para introduzir um grupo protector com o fim de impedir um dos dois grupos cetona de reagir com um reagente de Grignard, mas depois desta reacção o grupo tem de ser desbloqueado - estas operações acrescentam dois passos extra à síntese e baixam a economia atómica 
pos contribui, frequentemente de uma forma substancial, para uma perda de eficácia operacional da síntese e para a produção de uma elevada quantidade adicional de resíduos; por isso, no contexto da $\mathrm{QV}$, é muito importante que as vias de síntese sejam planificadas de forma a evitar, na medida do possível, a necessidade de usar grupos protectores.

\section{Da SV Ideal à Realidade da SV}

Quando presentemente se desenvolve uma nova via de síntese com o objectivo de optimizar a respectiva verdura, se todos os Doze Princípios da QV [13, 14] forem cabalmente cumpridos, a via materializará a chamada SV ideal. Assim, pode-se tentar definir as características essenciais da síntese ideal a partir daqueles Princípios, usando as respectivas metas (ver a Figura 5). Esta figura lista (seguir a seta) as características ideais de uma via de síntese, com respeito sucessivamente a:

(i) implementação química - simplicidade, etapa única (situação rara!), segurança química, rendimento total, máxima eficiência de átomos, ausência de desperdício de reagentes, etc.; e

(ii) efeitos ambientais - ambientalmente aceitável, reagentes renováveis, produto não tóxico, sem produção de resíduos e sem emissão de poluentes, etc.

Não se garante que a listagem de características da SV ideal apresentada na figura seja exaustiva (omite, por exemplo, os requisitos de energia), por isso se termina o percurso na estrela com um ponto de interrogação (?). As reacções de síntese, tal como as senhoras, são frequentemente sofisticadas e complexas - e, tal como a mulher ideal, a síntese ideal só existe na mente do químico verde, sendo difícil, se não impossível, encontrá-la na prática. No entanto, se porventura o for, é de esperar que tenha virtudes, ou vícios, insuspeitados, o que justifica que à interrogação se acrescente a exclamação ("?!"). Por exemplo, a figura exclui, embora seja óbvia se se pretender usar a síntese em Química Industrial, a necessidade de ela ser economicamente viável (o valor de venda do produto tem de cobrir os

\title{
CARACTERÍSTICAS DA SV IDEAL
}

\author{
CARACTERÍSTICAS \\ QUÍMICAS
}

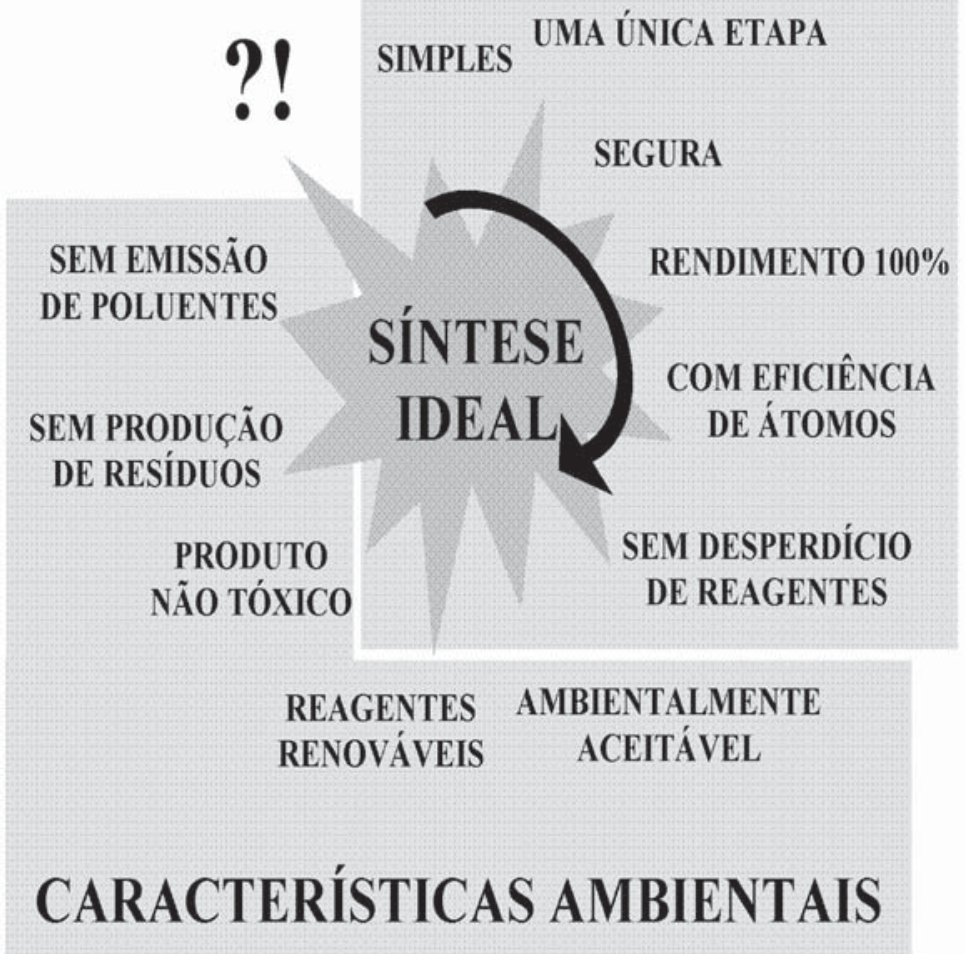

Figura 5 - Características da SV ideal: as características procuram traduzir os Doze Princípios da QV

custos dos reagentes e demais custos de produção, com margem positiva o lucro). Ora, um "vício terrível" que a grande maioria das vias de síntese académicas apresentam é não conseguirem ser realizadas à escala industrial sem dar prejuízos...Embora a apresentação do conceito de SV ideal feita na Figura 5 seja útil, já que permite a visualização global das metas a perseguir, na prática, dada a complexidade da química, não é possível atingi-las todas em pleno. Na realidade, a concepção da SV é bastante árdua, porque envolve a consideração simultânea de variados factores, referentes a todos os Doze Princípios, para todo o ciclo de vida do composto e do respectivo processo de síntese. Na Tabela 1 exemplifica-se a variedade de tais factores (não se pretende ser exaustivo, só se apresentou um por Princípio), usando o chamado enquadramento $\mathbf{R}_{4}$ [15], que vinca a necessidade de prestar atenção simultaneamente a Reacções, Resíduos, Reagentes e Recursos. A Tabela sugere bem a complexidade e dificuldades de design da SV para cumprir os Doze Princípios em globo.

É claro que existe uma forte interrelação entre a natureza das reacções químicas que podem ser usadas na via de síntese e a verdura desta, em particular, por exemplo, no que diz respeito às características químicas que permitem o percurso da via com bom aproveitamento no produto dos átomos dos reagentes. Quanto a este aspecto, aborda-se a seguir uma importante mudança de atitude dos químicos, que ocorreu há cerca de vinte anos e foi determinada pela sua crescente consciencialização sobre a importância do problema dos resíduos em Química Industrial - e que muito contribuiu para a génese da QV. 
Tabela 1 - Exemplos de aspectos a considerar na concepção da via para cumprir os Princípios e obter SV

\begin{tabular}{|c|c|}
\hline Aspecto & Princípio \\
\hline \multicolumn{2}{|l|}{ Reacções } \\
\hline Evitar o uso e produção de compostos tóxicos & 4 \\
\hline Realizar o controlo cuidadoso dos processos químicos & 11 \\
\hline Usar reacções seguras & 12 \\
\hline \multicolumn{2}{|l|}{ Resíduos } \\
\hline Evitar a formação de resíduos & 1 \\
\hline Maximizar a incorporação dos reagentes no produto & 2 \\
\hline Evitar derivatizações & 8 \\
\hline \multicolumn{2}{|l|}{ Reagentes } \\
\hline $\begin{array}{l}\text { Privilegiar o uso de catalisadores em detrimento de reagentes } \\
\text { estequiométricos }\end{array}$ & 9 \\
\hline Usar e conceber compostos inócuos & 3 \\
\hline Usar e conceber compostos degradáveis & 10 \\
\hline \multicolumn{2}{|l|}{ Recursos } \\
\hline Usar matérias-primas e reagentes renováveis & 7 \\
\hline Eliminar/minimizar solventes e substâncias auxiliares & 5 \\
\hline Minimizar os requisitos energéticos & 6 \\
\hline
\end{tabular}

\section{QUADRO 1}

\section{CONCEITOS DE AVALIAÇÃO DA UTILIZAÇÃO DOS ÁTOMOS NAS REACÇÕES}

ECONOMIA ATÓMICA

RAZÃO (\%) ENTRE A MASSA DE ÁTOMOS DOS REAGENTES ESTEQUIOMÉTRICOS INCORPORADOS NO PRODUTO E A MASSA TOTAL DE ÁTOMOS NOS REAGENTES

UTILIZAÇÃO ATÓMICA

RAZÃO (\%) ENTRE A MASSA DE PRODUTO E A SOMA DAS MASSAS DE TODAS AS SUBSTÂNCIAS PRODUZIDAS NA REACÇÃO (PRODUTO E COPRODUTOS)

\section{FACTOR E}

\section{RAZÃO (EM VALOR) ENTRE A MASSA DOS RESÍDUOS PRODUZIDOS E} A MASSA DO PRODUTO

\section{Mudança de Postura dos Químicos - Do DesPrezo PELOS Resíduos à QV}

No início dos anos noventa do século $X X$, os químicos orgânicos de síntese descobriram finalmente, após mais de cento e cinquenta anos de prática de inventar novas moléculas no laboratório ${ }^{4}$, que a arte da síntese consistia não só em obter o composto desejado, mas também em não obter resíduos. Por outras palavras, um objectivo importante que as reacções de síntese devem cumprir é incorporar os átomos aportados pelos reagentes na molécula do produto e não nas moléculas que se formam paralelamente a esta, as quais, em princípio, constituem resíduos.

Esta mudança de postura na visão das reacções de síntese, de reducionista para sistémica, resultou da introdução dos conceitos de economia atómica (Trost, 1991) [16], e de utilização atómica e factor $E$ (de "Environmental factor") (Sheldon, 1992) [17,18], que aliás contribuíram para formatar o Primeiro (Prevenção de resíduos) e Segundo (Economia atómica) dos Doze Princípios da QV, formulados poucos anos depois [13].

Estes conceitos são hoje muito importantes porque servem de base a métricas de massa, usadas cada vez mais na avaliação da verdura da química (ver, por exemplo, [19]), pelo que as suas definições são apresentadas no Quadro 1. Os dois primeiros conceitos avaliam a extensão da incorporação dos átomos dos reagentes no produto que se quer preparar - a meta da reacção.

Essa extensão deve ser a maior possível para que se perca a menor quantidade possível de átomos em resíduos - um objectivo tão importante como obter rendimento elevado. O factor $E$, cujo uso tem ganho importância crescente no domínio da química de síntese [20], ao comparar a massa de resíduos com a de produto, põe em evidência a extensão do problema da produção de resíduos no fabrico das substâncias químicas.

Estes novos conceitos de avaliação ("metrificação") das reacções químicas trouxeram consigo um modo inovador de olhar para o design da via de síntese, que passou a incluir a perseguição do embutimento no produto dos átomos proporcionados pelos reagentes a fim de minimizar a produção de resíduos.

A inovação pode ser expressa, em linguagem económica, em termos do conceito genérico de produtividade 5 : os químicos devem perseguir a produtividade dos átomos, um dos factores básicos que usam nas reacções químicas (outro é a energia). 
A introdução desta linguagem económica na química é um pouco mais recente que os conceitos de economia atómica e factor $E$ (o conceito de produtividade atómica deve-se a Steinbach e Winkerback, 2000) [21]. A relação da produtividade atómica com as métricas de massa referidas acima foi discutida com detalhe em [19]. O conceito de produtividade atómica é importante porque integra a SV no contexto mais global da desmaterialização, que, a par da diminuição da intensidade energética6 ("desenergialização"), constituem os pilares da eco-eficiência - constituindo uma ponte importante entre a QV e a Ecologia Industrial.

\section{Conclusões}

A discussão anterior sugere que o ensino da química deve dar mais atenção ao conceito de via de síntese e, em particular, ao de SV, cujo design é complexo (e, frequentemente, elusivo), o que acarreta que a sua prática seja afinal problemática e custosa, exigindo uma mudança de atitude mental dos químicos no sentido de adquirirem uma postura sistémica. Esta mudança está implícita nos conceitos de economia atómica e utilização atómica, devidos respectivamente a Trost e Sheldon, que constituem "saltos em frente" inovatórios de grande calibre quanto à visão que os químicos devem ter da síntese orgânica. Este facto merece ser realçado, já que as mudanças de paradigma são sempre difíceis de concretizar.

No artigo seguinte desta série discutirse-á como a prática histórica da síntese em Química Industrial evidencia que, em variadas situações, esta introduziu vias de síntese com características verdes de raiz; e que vários processos químico-industriais foram sendo depois aperfeiçoados no sentido de perseguir o aumento da verdura presentemente embutida na ideia de SV ideal. Esta antecipação de conclusões do artigo seguinte, embora seja prematura, é incluída aqui para terminar este com uma perspectiva optimista: a SV pode ser complexa e difícil, mas a história mostra que, se os químicos lutarem por ela, podem atingi-la em grau elevado.
Notas

1 Os livros [1-2], pequenos textos com menos de 100 páginas, são úteis para os químicos não profissionais da síntese e professores que quiserem obter uma ideia global da importância e problemas do escalamento da via de síntese.

2 Por exemplo, duas reacções sucessivas que envolvam um intermediário transiente, não isolável, constituem apenas um único passo.

3 Chama-se síntese total a um processo de síntese realizado totalmente por via química, em oposição a processos de síntese em que se parte de um produto natural isolado a partir da natureza, portanto de origem biológica, cuja molécula é modificada por reacções químicas para obter outro composto neste caso, o composto é obtido por síntese parcial.

4 A primeira síntese de uma molécula orgânica foi realizada em 1828 , pelo químico alemão $\mathrm{F}$. Wohler (1800-1882) - a ureia, $\left(\mathrm{NH}_{2}\right)_{2} \mathrm{CO}$; esta experiência marca o nascimento da Química Orgânica. O marco seguinte desta foi a síntese do primeiro composto orgânico artificial ("sintético"), o corante malvina, por W. H. Perkin (1856), quando pretendia sintetizar o quinino.

5 A produtividade de um factor de produção é a razão entre quantidade de produto e a quantidade de factor usado, por exemplo, é bem conhecida a produtividade do trabalho.

6 A intensidade energética é a razão da energia dispendida por unidade de valor dos bens produzidos, por exemplo, para uma economia nacional é a razão entre a energia total gasta pelo país e o respectivo Produto Interno Bruto (na base anual).

\section{REFERÊNCIAS}

[1] S. Lee e G. Robinson, Process Development - Fine Chemicals from Gram to Kilograms, Oxford, 1995.

[2] J. H. Atherton e K. J. Carpenter, Process Development: Physicochemical Concepts, Oxford, 1999.

[3] N. G. Anderson, Practical Process Research \& Development, Academic Press, 2000.
[4] W. Cabri e R. Di Fabio, From Bench to Market - The Evolution of Chemical Synthesis, Oxford, 2000.

[5] G. H. Vogel, Process Development From the Initial Idea to the Chemical Production Plant, Wiley-VCH, 2005.

[6] D. Walker, The Management of Chemical Process Development in the Pharmaceutical Industry, Wiley-Interscience, 2008.

[7] M. Nafissi, J. A. Ragan e K. M. DeVries, "From Bench to Pilot Plant - Process Research in the Pharmaceuticallndustry",ASCSymp. S.817(2002).

[8] A. F. Abdel-Magid e J. A. Ragan, Chemical Process Research - The Art of Practical Organic Synthesis", ACS Symp. S. 870 (2004).

[9] M. Zlokarnik, Scale-up in Chemical Engineering, Wiley-VCH, 2002.

[10] G. B. Tatterson, Process Scaleup and Design, NC A\&T State University, 2002.

[11] D. J. C. Constable, A. D. Curzons, L. M. F. Santos, G. R. Geen, R. E. Hannah, J. D. Hayler, J. Kitteringham, M. A. McGuire, J. E. Richardson, P. Smith, R. L. Webb e M. Yu, "Green Chemistry Measures for Process Research and Development", Green Chem. 3 (2001) 7-9.

[12] M. C. Cann e M. E. Connelly, RealWorld Cases in Green Chemistry, ACS, 2000, p. 9.

[13] P. T. Anastas e J. C. Warner, Green Chemistry - Theory and Practice, Oxford UP, 1998, p. 30.

[14] A. A. S. C. Machado, "Química e Desenvolvimento Sustentável - QV, QUIVES, QUISUS", Química - Bol. S. P. Q. 95 (2004) 59-67.

[15] X. Domènech, Química Verde, Rubes, 2005, p. 46.

[16] B. M. Trost, "The Atom Economy A Search for Synthetic Efficiency", Science 254 (1991) 1471-1477.

[17] R. A. Sheldon, "Organic Synthesis Past, Present and Future", Chem. Ind. (London) (1992) 903-906.

[18] R. A. Sheldon, "Consider the Environmental Quotient", ChemTech 24 (3) (1994) 39-47.

[19] A. A. S. C. Machado, "Métricas da Química Verde - A Produtividade Atómica", Química - Bol. S. P. Q. 107 (2007) 47-55.

[20] R. A. Sheldon, "The Factor F: Fifteen Years On”, Green Chem. 9 (2007) 1273-1283.

[21] A. Steinbach e R. Winkerbach, "Choose Products for Their Productivity", Chem. Eng. (2000, Abril) 94-104. 